\title{
UNIQUENESS OF SOLUTIONS TO SCHRÖDINGER EQUATIONS ON $H$-TYPE GROUPS
}

\author{
SALEM BEN SAÏD ${ }^{\bowtie}$, SUNDARAM THANGAVELU and VENKU NAIDU DOGGA
}

(Received 4 June 2011; accepted 19 May 2013; first published online 7 August 2013)

Communicated by M. Cowling

\begin{abstract}
This paper deals with the Schrödinger equation $i \partial_{s} u(\mathbf{z}, t ; s)-\mathcal{L} u(\mathbf{z}, t ; s)=0$, where $\mathcal{L}$ is the subLaplacian on the Heisenberg group. Assume that the initial data $f$ satisfies $|f(\mathbf{z}, t)| \lesssim q_{\alpha}(\mathbf{z}, t)$, where $q_{s}$ is the heat kernel associated to $\mathcal{L}$. If in addition $\left|u\left(\mathbf{z}, t ; s_{0}\right)\right| \lesssim q_{\beta}(\mathbf{z}, t)$, for some $s_{0} \in \mathbb{R} \backslash\{0\}$, then we prove that $u(\mathbf{z}, t ; s)=0$ for all $s \in \mathbb{R}$ whenever $\alpha \beta<s_{0}^{2}$. This result holds true in the more general context of $H$-type groups. We also prove an analogous result for the Grushin operator on $\mathbb{R}^{n+1}$.
\end{abstract}

2010 Mathematics subject classification: primary 22E30; secondary 43B30, 43A80.

Keywords and phrases: $H$-type groups, sub-Laplacian, Schrödinger equation, heat kernel, spherical harmonics.

\section{Introduction}

Consider the solution $u(x, s)$ of the Schrödinger equation

$$
\left\{\begin{array}{l}
i \partial_{s} u(x, s)=\Delta u(x, s), \quad(x, s) \in \mathbb{R}^{n} \times \mathbb{R} \\
u(x, 0)=f(x) .
\end{array}\right.
$$

In [2] Chanillo has shown that if the initial condition $f$ has certain Gaussian decay then the solution $u(x, s)$ at a later time cannot have an arbitrary Gaussian decay. This is reminiscent of the Euclidean Hardy theorem, which states that a function $f$ and its Fourier transform $\widehat{f}$ cannot have arbitrary Gaussian decay. More precisely, if the definition of the Fourier transform is taken as

$$
\widehat{f}(\xi)=(2 \pi)^{-n / 2} \int_{\mathbb{R}^{n}} f(x) e^{-i x \cdot \xi} d x,
$$

then the conditions

$$
|f(x)| \lesssim e^{-\alpha|x|^{2}}, \quad|\widehat{f}(\xi)| \lesssim e^{-\beta|\xi|^{2}}
$$

The work of the last two authors is supported by a J. C. Bose Fellowship from DST, India. (c) 2013 Australian Mathematical Publishing Association Inc. 1446-7887/2013 \$16.00 
can be satisfied for a nontrivial $f$ only if $\alpha \beta \leq 1 / 4$. This can be viewed as an uncertainty principle for the Fourier transform. The notation $X \lesssim Y$ is (and will be) used to indicate that $X \leq C Y$ with a positive constant $C$ independent of significant quantities.

Since the solution of the aforementioned Schrödinger equation can be expressed in terms of the Fourier transform of $f$, by a straightforward application of Hardy's theorem, Chanillo obtained the following uniqueness theorem for solutions of the Schrödinger equation.

THeorem 1.1 (see [2]). Let $u(x, s)$ be the solution of the Schrödinger equation (1.1), where $f$ is assumed to satisfy the estimate $|f(x)| \lesssim e^{-\alpha|x|^{2}}$ for some positive constant $\alpha$. If at a later time $s=s_{0}$ the solution satisfies $\left|u\left(x, s_{0}\right)\right| \lesssim e^{-\beta|x|^{2}}$ for some positive constant $\beta$, then $f=0$ whenever $\alpha \beta<s_{0}^{2}$.

Hardy's theorem as stated above goes back to the work of Hardy in 1933 and later similar results for the Fourier transforms on Lie groups have been established, see [17]. However, until the work of Chanillo, Hardy-type theorems were considered only in the context of heat equations, and his work triggered a lot of attention on Schrödinger evolutions. Chanillo himself treated the Schrödinger equation on complex Lie groups where the initial condition was assumed to be $K$-biinvariant [2]. However, if we use Radon transform, the problem can be reduced to the Euclidean case and his result holds without any restriction either on the group or on the initial condition. Recently, somewhat more precise results of this kind have been proved by Pasquale and Sundari [12] in the context of symmetric spaces.

Similar uniqueness results for other Schrödinger evolutions and for the Kortewegde Vries equation have received a good deal of attention in recent years (see, for instance, $[4,5,8,10,15,19])$. These authors have developed powerful PDE techniques to deal with uniqueness results. Completing a full circle, in a recent work Cowling et al. [3] have used a uniqueness theorem for the Schrödinger equation to give a 'real variable proof' of Hardy's theorem. See also the works [6,7], where the authors deal with equations with nonconstant lower order terms and/or nonlinear equations.

In this paper we are interested in proving an analogue of Chanillo's theorem for $H$-type groups. Let $G$ be such a group and denote by $\mathcal{L}_{G}$ the corresponding subLaplacian. We consider the following initial value problem for the Schrödinger equation associated with $\mathcal{L}_{G}$ :

$$
\left\{\begin{array}{l}
i \partial_{s} u(g, s)-\mathcal{L}_{G} u(g, s)=0, \quad g \in G, s \in \mathbb{R} \\
u(g, 0)=f(g),
\end{array}\right.
$$

where $f$ is assumed to be in $L^{2}(G)$. Our goal is to find sufficient conditions on the behavior of the solution $u$ at two different times $s=0$ and $s=s_{0}$ which guarantee that $u \equiv 0$ is the unique solution to (1.2).

We write the elements of $G$ as $g=(\mathbf{v}, \mathbf{t})$, where $\mathbf{t}$ comes from the center of $G$. We denote by $h_{\alpha}(\mathbf{v}, \mathbf{t})$ the heat kernel associated to the sub-Laplacian $\mathcal{L}_{G}$. We prove the following theorem. 
Theorem 1.2. Let $u(\mathbf{v}, \mathbf{t} ; s)$ be the solution of the Schrödinger equation (1.2). Assume that $|f(\mathbf{v}, \mathbf{t})| \lesssim h_{\alpha}(\mathbf{v}, \mathbf{t})$ for some $\alpha>0$. Further, suppose that there exists $s_{0} \in \mathbb{R} \backslash\{0\}$ such that $\left|u\left(\mathbf{v}, \mathbf{t} ; s_{0}\right)\right| \lesssim h_{\beta}(\mathbf{v}, \mathbf{t})$ for some $\beta>0$. If $\alpha \beta<s_{0}^{2}$ then $u(\mathbf{v}, \mathbf{t} ; s)=0$ for all $(\mathbf{v}, \mathbf{t}) \in G$ and for all $s \in \mathbb{R}$.

Our approach uses Hardy's theorem for the Hankel transform obtained in [18]. The theorem says that a function and its Hankel transform both cannot have arbitrary Gaussian decay at infinity unless the function is identically zero. It is interesting to note that our method does not use Hardy's theorem for the Heisenberg group proved in [17].

The $(2 n+1)$-dimensional Heisenberg group, denoted by $\mathbb{H}^{n}$, is the most wellknown example of an $H$-type group. In Section 3 we prove Theorem 1.2 for the case $G=\mathbb{H}^{n}$. Once the theorem is proved for $\mathbb{H}^{n}$ it is not difficult to extend the proof for an arbitrary $H$-type group. This class of groups was introduced by Kaplan in [9] and the list of $H$-type groups includes the so-called Heisenberg groups and their analogues built-up with quaternions or octonions in place of complex numbers, as well as many other groups.

We also prove an analogue of the above theorem for the Grushin operator $\mathcal{G}=$ $-\Delta_{\mathbb{R}^{n}}-|x|^{2} \partial_{t}^{2}$ on $\mathbb{R}^{n+1}$. The behavior of this operator is very similar to that of the subLaplacian $\mathcal{L}_{\mathbb{H}^{n}}$ as can be easily seen by comparing the explicit expression for the latter with the above when $n$ is even. The spectral decomposition of $\mathcal{G}$ is explicitly known and we also have a good knowledge of the associated heat kernel. Let $\kappa_{s}\left(x, y, t-t^{\prime}\right)$ denote the heat kernel so that

$$
u(x, t ; s):=\int_{\mathbb{R}^{n+1}} \kappa_{s}\left(x, y, t-t^{\prime}\right) f\left(y, t^{\prime}\right) d y d t^{\prime}, \quad(x, t) \in \mathbb{R}^{n+1}, s \in \mathbb{R}
$$

solves the heat equation associated to the Grushin operator $\mathcal{G}$. The following is the analogue of Theorem 1.2 for the Grushin operator.

Theorem 1.3. Let $u(x, t ; s)$ be the solution of the Schrödinger equation associated to the Grushin operator $\mathcal{G}=-\Delta_{\mathbb{R}^{n}}-|x|^{2} \partial_{t}^{2}$, with initial condition $f \in L^{2}\left(\mathbb{R}^{n+1}\right)$. Suppose that

$$
\begin{gathered}
|f(x, t)| \lesssim \kappa_{\alpha}(x, 0, t) \\
\left|u\left(x, t ; s_{0}\right)\right| \lesssim \kappa_{\beta}(x, 0, t),
\end{gathered}
$$

for some $\alpha, \beta>0$ and for a fixed $s_{0} \in \mathbb{R} \backslash\{0\}$. Then $u(x, t ; s)=0$ on $\mathbb{R}^{n+1} \times \mathbb{R}$ whenever $\alpha \beta<s_{0}^{2}$.

We indicate a proof of this theorem in Section 5. As we mentioned above, the proof of the main Theorem 1.2 for the case $G=\mathbb{H}^{n}$ uses an analogue of Hardy's theorem for the Hankel transform. An important role is played by a Hecke-Bochner type formula for the special Hermite projections in reducing the problem to the Euclidean setup. The proof can also be carried out for Grushin operators, which are very similar to the sub-Laplacians, thanks to an analogue of the Hecke-Bochner formula for Hermite 
projection operators. In the last section we briefly indicate how other versions of our main result can be proved for the Heisenberg group $\mathbb{H}^{n}$.

Note added in proof. After this paper has been submitted for publication, a related preprint was produced by Ludwig and Müller [11], where the authors generalize our main result to every step two nilpotent Lie group.

\section{Background}

The $(2 n+1)$-dimensional Heisenberg group, denoted by $\mathbb{H}^{n}$, is $\mathbb{C}^{n} \times \mathbb{R}$ equipped with the group law

$$
(\mathbf{z}, t)(\mathbf{w}, s)=\left(\mathbf{z}+\mathbf{w}, t+s+\frac{1}{2} \operatorname{Im}(\mathbf{z} \cdot \overline{\mathbf{w}})\right) .
$$

Under this multiplication $\mathbb{H}^{n}$ becomes a nilpotent unimodular Lie group, the Haar measure being the Lebesgue measure $d \mathbf{z} d t$ on $\mathbb{C}^{n} \times \mathbb{R}$. The corresponding Lie algebra is generated by the vector fields

$$
X_{j}:=\frac{\partial}{\partial x_{j}}+\frac{1}{2} y_{j} \frac{\partial}{\partial t}, \quad Y_{j}:=\frac{\partial}{\partial y_{j}}-\frac{1}{2} x_{j} \frac{\partial}{\partial t},
$$

for $j=1,2, \ldots, n$, and by $T:=\frac{\partial}{\partial t}$. The sub-Laplacian

$$
\mathcal{L}_{\mathbb{H}^{n}}:=-\sum_{j=1}^{n} X_{j}^{2}+Y_{j}^{2}
$$

can be written as

$$
\mathcal{L}_{\mathbb{H}^{n}}=-\Delta_{\mathbb{R}^{2 n}}-\frac{1}{4}|\mathbf{z}|^{2} \frac{\partial^{2}}{\partial t^{2}}+\sum_{j=1}^{n}\left(x_{j} \frac{\partial}{\partial y_{j}}-y_{j} \frac{\partial}{\partial x_{j}}\right) \frac{\partial}{\partial t} .
$$

Henceforth we will denote the sub-Laplacian $\mathcal{L}_{\mathbb{H}^{n}}$ by $\mathcal{L}$. This second-order differential operator $\mathcal{L}$ is hypoelliptic, self-adjoint and nonnegative. It generates a semigroup with kernel $q_{s}(\mathbf{z}, t)$, called the heat kernel. In particular, $q_{s}(\mathbf{z}, t)$ is nonnegative and has the property

$$
q_{r^{2} s}(\mathbf{z}, t)=r^{-2(n+1)} q_{s}\left(r^{-1} \mathbf{z}, r^{-2} t\right), \quad \forall r \neq 0 .
$$

Moreover,

$$
\int_{\mathbb{R}} e^{i \lambda t} q_{s}(\mathbf{z}, t) d t=(4 \pi)^{-n}\left(\frac{\lambda}{\sinh \lambda s}\right)^{n} e^{-\frac{1}{4} \lambda(\operatorname{coth} s \lambda)|\mathbf{z}|^{2}}
$$

(see [17]). Henceforth, for $f \in L^{1}\left(\mathbb{H}^{n}\right)$ and $\lambda \in \mathbb{R}$, we will write

$$
f^{\lambda}(\mathbf{z}):=\int_{\mathbb{R}} e^{i \lambda t} f(\mathbf{z}, t) d t
$$

to denote the inverse Fourier transform of $f(\mathbf{z}, t)$ in the $t$-variable. 
Let $f$ and $g$ be two functions on $\mathbb{H}^{n}$. The convolution of $f$ with $g$ is defined by

$$
(f * g)(\mathbf{z}, t)=\int_{\mathbb{H}^{n}} f((\mathbf{z}, t)(-\mathbf{w}, s)) g(\mathbf{w}, s) d \mathbf{w} d s .
$$

An easy calculation shows that

$$
(f * g)^{\lambda}(\mathbf{z})=\int_{\mathbb{C}^{n}} f^{\lambda}(\mathbf{z}-\mathbf{w}) g^{\lambda}(\mathbf{w}) e^{i(\lambda / 2) \operatorname{Im}(\mathbf{z} \cdot \overline{\mathbf{w}})} d \mathbf{w} .
$$

The integral on the right-hand side is called the $\lambda$-twisted convolution of $f^{\lambda}$ with $g^{\lambda}$, and will be denoted by $f^{\lambda} *_{\lambda} g^{\lambda}$.

Now we pin down some properties of the heat kernel $q_{s}(\mathbf{z}, t)$.

FАСт 2.1. The heat kernel satisfies the semigroup property $q_{\alpha} * q_{\beta}(\mathbf{z}, t)=q_{\alpha+\beta}(\mathbf{z}, t)$.

The following is a slight modification of [17, Proposition 2.8.2].

FАст 2.2. The heat kernel $q_{s}(\mathbf{z}, t)$ satisfies the following estimate

$$
q_{s}(\mathbf{z}, t) \lesssim s^{-n-1} e^{-(\pi / 2)(|t| / s)} e^{-(1 / 4)\left(|\mathbf{z}|^{2} / s\right)}, \quad s>0 .
$$

Indeed, by [17, (2.8.9) and (2.8.10)],

$$
q_{1}(\mathbf{z}, t) \lesssim e^{-(\pi / 2)|t|} e^{-(1 / 4)|\mathbf{z}|^{2}}
$$

Now (2.3) follows from the fact that $q_{s}(\mathbf{z}, t)=s^{-n-1} q_{1}\left(s^{-1 / 2} \mathbf{z}, s^{-1} t\right)$ for all $s>0$.

For $\alpha \in \mathbb{N}_{0}^{n}$ and $\mathbf{z} \in \mathbb{C}^{n}$, a monomial in the variables $z_{1}, \ldots, z_{n}$ is a product $\mathbf{z}^{\alpha}=$ $z_{1}^{\alpha_{1}} \cdots z_{n}^{\alpha_{n}}$. The number $|\alpha|$ is called the total degree of $\mathbf{z}^{\alpha}$. Let $\mathcal{P}$ be the set of all polynomials of the form $P(\mathbf{z})=\sum_{|\alpha|+|\beta| \leq m} a_{\alpha, \beta} \mathbf{z}^{\alpha} \overline{\mathbf{z}}^{\beta}$. For each pair of nonnegative integers $(p, q)$, we define $\mathcal{P}_{p, q}$ to be the subspace of $\mathcal{P}$ consisting of all polynomials of the form $P(\mathbf{z})=\sum_{|\alpha|=p} \sum_{|\beta|=q} a_{\alpha, \beta} \mathbf{z}^{\alpha} \overline{\mathbf{z}}^{\beta}$.

Let $\mathcal{H}_{p, q}:=\left\{P \in \mathcal{P}_{p, q} \mid \Delta P=0\right\}$, where $\Delta$ denotes the Laplacian on $\mathbb{C}^{n}$. The elements of $\mathcal{H}_{p, q}$ are called bigraded solid harmonics of degree $(p, q)$. We will denote by $\mathcal{S}_{p, q}$ the space of all restrictions of bigraded solid harmonics of degree $(p, q)$ to the sphere $S^{2 n-1}$. By [17], the space $L^{2}\left(S^{2 n-1}\right)$ is the orthogonal direct sum of the spaces $\mathcal{S}_{p, q}$, with $p, q \geq 0$. We choose an orthonormal basis $\left\{Y_{p, q}^{j} \mid 1 \leq j \leq d(p, q)\right\}$ for $\mathcal{S}_{p, q}$. Then by standard arguments it follows that every continuous function $\psi$ on $\mathbb{C}^{n}$ can be expanded as

$$
\psi(r \omega)=\sum_{p, q \geq 0} \sum_{j=1}^{d(p, q)} C_{p, q}^{j}(\psi)(r) Y_{p, q}^{j}(\omega), \quad r>0, \omega \in S^{2 n-1}
$$

where

$$
C_{p, q}^{j}(\psi)(r):=\int_{S^{2 n-1}} \psi(r \omega) \overline{Y_{p, q}^{j}(\omega)} d \sigma(\omega)
$$


For $k \in \mathbb{N}$, we write $L_{k}^{(n-1)}$ for the Laguerre polynomial defined by

$$
L_{k}^{(n-1)}(t)=\sum_{j=0}^{k} \frac{(-1)^{j} \Gamma(n+k)}{(k-j) ! \Gamma(n+j)} t^{j} .
$$

For $\lambda \in \mathbb{R}^{*}$, define the Laguerre function $\varphi_{k, \lambda}^{(n-1)}$ by

$$
\varphi_{k, \lambda}^{(n-1)}(\mathbf{z})=e^{-(|\lambda| / 4)|\mathbf{z}|^{2}} L_{k}^{(n-1)}\left(\frac{|\lambda|}{2}|\mathbf{z}|^{2}\right)
$$

for $\mathbf{z} \in \mathbb{C}^{n}$. Suppose that $\psi$ is a radial function in $L^{1}\left(\mathbb{C}^{n}\right)$. Then the function $F$ defined by $F(|\mathbf{z}|)=\psi(\mathbf{z})$ is in $L^{1}\left(\mathbb{R}^{+}, r^{2 n-1} d r\right)$. For the following Hecke-Bochner formula we refer to [17, Theorem 2.6.1].

Theorem 2.3. Consider $\psi(\mathbf{z})=P(\mathbf{z}) F(|\mathbf{z}|)$, where $P \in \mathcal{H}_{p, q}$ and $F \in L^{1}\left(\mathbb{R}^{+}, r^{2 n-1} d r\right)$. Then for $\lambda \in \mathbb{R}^{*}$,

$$
\psi *_{\lambda} \varphi_{k, \lambda}^{(n-1)}(\mathbf{z})=(2 \pi)^{-n}|\lambda|^{p+q} P(\mathbf{z})\left\{F *_{\lambda} \varphi_{k-p, \lambda}^{(n+p+q-1)}\right\}(\mathbf{z}),
$$

where the convolution on the right-hand side is taken on $\mathbb{C}^{n+p+q}$ treating the radial functions $F$ and $\varphi_{k-p, \lambda}^{(n+p+q-1)}$ as functions on $\mathbb{C}^{n+p+q}$. More explicitly,

$$
\begin{aligned}
F *_{\lambda} & \varphi_{k-p, \lambda}^{(n+p+q-1)}(\mathbf{z}) \\
= & \frac{(2 \pi)^{n+p+q}|\lambda|^{(n+p+q) / 2} 2^{-(n+p+q)+1} \Gamma(k-p+1)}{\Gamma(k+n+q)} \varphi_{k-p, \lambda}^{(n+p+q-1)}(\mathbf{z}) \\
& \times\left(\int_{0}^{\infty} g(s) L_{k-p}^{(n+p+q-1)}\left(\frac{|\lambda|}{2} s^{2}\right) e^{-(|\lambda| / 4) s^{2}} s^{2(n+p+q)-1} d s\right) .
\end{aligned}
$$

To end this section, let us recall Hardy's uncertainty principle for the Hankel transform. For $v>-1 / 2$ and for a Schwartz function $F$ on $\mathbb{R}^{+}$, the Hankel transform of order $v$ is defined by

$$
\mathscr{H}_{\nu} F(s)=2^{-v} \int_{0}^{\infty} F(r) J_{v}(r s) r^{2 v+1} d r,
$$

where $J_{v}(w)$ is the normalized Bessel function of order $v$ defined by

$$
J_{v}(w)=\sum_{k=0}^{\infty} \frac{(-1)^{k}\left(\frac{w}{2}\right)^{2 k}}{k ! \Gamma(v+k+1)} .
$$

Theorem 2.4 [18, Hardy's theorem]. Let $F$ be a measurable function on $\mathbb{R}^{+}$such that

$$
F(r)=O\left(e^{-\alpha r^{2}}\right), \quad \mathscr{H}_{v} F(s)=O\left(e^{-\beta s^{2}}\right),
$$

for some positive $\alpha$ and $\beta$. Then $F=0$ whenever $\alpha \beta>1 / 4$ and $F(r)=C e^{-\alpha r^{2}}$ whenever $\alpha \beta=1 / 4$. 


\section{Schrödinger equation on $\mathbb{H}^{n} \times \mathbb{R}$}

Let us consider the Schrödinger equation on $\mathbb{H}^{n} \times \mathbb{R}$

$$
\left\{\begin{array}{l}
i \partial_{s} u(\mathbf{z}, t ; s)=\mathcal{L} u(\mathbf{z}, t ; s), \quad(\mathbf{z}, t) \in \mathbb{H}^{n}, s \in \mathbb{R} \\
u(\mathbf{z}, t ; 0)=f(\mathbf{z}, t) .
\end{array}\right.
$$

As the closure of $\mathcal{L}$ on $C_{c}^{\infty}\left(\mathbb{H}^{n}\right)$ is a self-adjoint operator, $-i \mathcal{L}$ generates a unitary semigroup $e^{-i s \mathcal{L}}$ on $L^{2}\left(\mathbb{H}^{n}\right)$, and the solution of the above Schrödinger equation is given by

$$
u(\mathbf{z}, t ; s)=e^{-i s \mathcal{L}} f(\mathbf{z}, t) .
$$

Theorem 3.1. Let $u(\mathbf{z}, t ; s)$ be the solution to the Schrödinger equation (3.1). Suppose that

$$
\begin{array}{r}
|f(\mathbf{z}, t)| \lesssim q_{\alpha}(\mathbf{z}, t), \\
\left|u\left(\mathbf{z}, t ; s_{0}\right)\right| \lesssim q_{\beta}(\mathbf{z}, t),
\end{array}
$$

for some $\alpha, \beta>0$ and for a fixed $s_{0} \in \mathbb{R}^{*}$. Then $u(\mathbf{z}, t ; s)=0$ on $\mathbb{H}^{n} \times \mathbb{R}$ whenever $\alpha \beta<s_{0}^{2}$.

The remaining part of this section is devoted to the proof of the above statement.

The heat kernel $q_{s}(\mathbf{z}, t)$ has an analytic continuation in $s$ as long as the real part of $s$ is positive. However, due to the zeros of the sine function, it follows from formula (2.1) for $q_{s}^{\lambda}(\mathbf{z})$ that the kernel $q_{i s}(\mathbf{z}, t)$ does not exist. Hence the solution $u(\mathbf{z}, t ; s)$ does not have an integral representation. To counter this difficulty we will consider the following regularized problem on $\mathbb{H}^{n} \times \mathbb{R}$ :

$$
\left\{\begin{array}{l}
i \partial_{s} u_{\epsilon}(\mathbf{z}, t ; s)=\mathcal{L} u_{\epsilon}(\mathbf{z}, t ; s), \quad \epsilon>0 \\
u_{\epsilon}(\mathbf{z}, t ; 0)=f_{\epsilon}(\mathbf{z}, t)
\end{array}\right.
$$

where $f_{\epsilon}(\mathbf{z}, t):=e^{-\epsilon \mathcal{L}} f(\mathbf{z}, t)$. The solution $u_{\epsilon}$ on $\mathbb{H}^{n} \times \mathbb{R}$ is given by

$$
u_{\epsilon}(\mathbf{z}, t ; s)=e^{-i s \mathcal{L}} f_{\epsilon}(\mathbf{z}, t)=f * q_{\zeta}(\mathbf{z}, t),
$$

with $\zeta=\epsilon+i$ and

$$
q_{\zeta}(\mathbf{z}, t):=\frac{1}{\left(8 \pi^{2}\right)^{n}} \int_{\mathbb{R}} e^{-i \lambda t}\left(\frac{\lambda}{\sinh \lambda \zeta}\right)^{n} e^{-\frac{1}{4} \lambda(\operatorname{coth} \zeta \lambda)|\mathbf{z}|^{2}} d \lambda .
$$

Observe that the kernel $q_{\zeta}(\mathbf{z}, t)$ is well defined.

LEMMA 3.2. Under the assumptions (3.1a) and (3.1b),

$$
\begin{array}{r}
\left|f_{\epsilon}(\mathbf{z}, t)\right| \lesssim q_{\alpha+\epsilon}(\mathbf{z}, t), \\
\left|u_{\epsilon}\left(\mathbf{z}, t ; s_{0}\right)\right| \lesssim q_{\beta+\epsilon}(\mathbf{z}, t) .
\end{array}
$$


Proof. For the first estimate,

$$
\begin{aligned}
\left|f_{\epsilon}(\mathbf{z}, t)\right|=\left|e^{-\epsilon \mathcal{L}} f(\mathbf{z}, t)\right| & =\left|f * q_{\epsilon}(\mathbf{z}, t)\right| \\
& \lesssim q_{\alpha+\epsilon}(\mathbf{z}, t) .
\end{aligned}
$$

Above we have used Fact 2.1 and that $q_{s}$ is nonnegative. Similarly,

$$
\begin{aligned}
\left|u_{\epsilon}\left(\mathbf{z}, t ; s_{0}\right)\right| & =\left|u\left(\cdot, \cdot ; s_{0}\right) * q_{\epsilon}(\mathbf{z}, t)\right| \\
& \lesssim q_{\beta+\epsilon}(\mathbf{z}, t) .
\end{aligned}
$$

Recall that for $\lambda \in \mathbb{R}$, the notation $f^{\lambda}(\mathbf{z})$ stands for the inverse Fourier transform of $f(\mathbf{z}, t)$ in the $t$-variable. In view of the hypothesis (3.1a) on $f$ and the estimate (2.3) on the heat kernel, one can see that the function $\lambda \mapsto f^{\lambda}(\mathbf{z})$ extends to a holomorphic function of $\lambda$ on the strip $|\operatorname{Im}(\lambda)|<\pi / 2 \alpha$. Thus the following statement holds.

Lemma 3.3. Under the hypothesis (3.1a) on $f$, the inverse Fourier transform $f^{\lambda}(\mathbf{z})$ of $f(\mathbf{z}, t)$ in the $t$-variable extends to a holomorphic function of $\lambda$ in a tubular neighborhood in $\mathbb{C}$ of the real line.

We point out that the above lemma also holds for the function $\lambda \mapsto f_{\epsilon}^{\lambda}$.

StRategy. To prove Theorem 3.1 it is enough to show that $f=0$ on $\mathbb{H}^{n}$ whenever $\alpha \beta<s_{0}^{2}$. Note that, by Lemma 3.3, showing that $f^{\lambda}=0$ on $\mathbb{C}^{n}$ for $0<\lambda<\delta$, for some $\delta>0$, will force $f^{\lambda}=0$ on $\mathbb{C}^{n}$ for all $\lambda \in \mathbb{R}$, and therefore $f=0$ on $\mathbb{H}^{n}$. Further, since $f_{\epsilon}^{\lambda}=f^{\lambda} *_{\lambda} q_{\epsilon}^{\lambda}$, then proving that $f^{\lambda}=0$ on $\mathbb{C}^{n}$ for $0<\lambda<\delta$ is equivalent to showing the same for $f_{\epsilon}^{\lambda}$. Now, to prove that $f_{\epsilon}^{\lambda}(\mathbf{z})=0$ for $0<\lambda<\delta$, for some $\delta>0$, it is enough to prove that the spherical harmonic coefficients

$$
C_{p, q}^{j}\left(f_{\epsilon}^{\lambda}\right)(r)=\int_{S^{2 n-1}} f_{\epsilon}^{\lambda}(r \omega) \overline{Y_{p, q}^{j}(\omega)} d \sigma(\omega)
$$

vanish for $0<\lambda<\delta$, for all $p, q \geq 0$ and for all $1 \leq j \leq d(p, q)$. In conclusion, the proof of Theorem 3.1 reduces to prove that if $\alpha \beta<s_{0}^{2}$ then $C_{p, q}^{j}\left(f_{\epsilon}^{\lambda}\right)=0$ on $\mathbb{R}^{+}$for $0<\lambda<\delta$, for all $p, q \geq 0$ and for all $1 \leq j \leq d(p, q)$.

The following theorem will be of crucial importance to us.

Theorem 3.4. Let us fix $p_{0}, q_{0} \geq 0$ and $1 \leq j_{0} \leq d\left(p_{0}, q_{0}\right)$. For all $r>0$, there exists $a$ constant $c_{\lambda}$ which depends only on $\lambda$ such that

$$
\begin{gathered}
\int_{S^{2 n-1}} u_{\epsilon}^{\lambda}\left(r \omega ; s_{0}\right) \overline{Y_{p_{0}, q_{0}}^{j_{0}}(\omega)} d \sigma(\omega)=c_{\lambda} r^{p_{0}+q_{0}} e^{i(\lambda / 4) r^{2} \operatorname{cotg}\left(\lambda s_{0}\right)} \\
\times \mathscr{H}_{n+p_{0}+q_{0}-1}\left(e^{i(\lambda / 4)(\cdot)^{2} \operatorname{cotg}\left(\lambda s_{0}\right)} \widetilde{C}_{p_{0}, q_{0}}^{j_{0}}\left(f_{\epsilon}^{\lambda}\right)\right)\left(\frac{\lambda r}{2 \sin \left(\lambda s_{0}\right)}\right)
\end{gathered}
$$

where $u_{\epsilon}^{\lambda}\left(\mathbf{z} ; s_{0}\right)$ denotes the inverse Fourier transform of $u_{\epsilon}\left(\mathbf{z}, t ; s_{0}\right)$ in the $t$-variable, $\mathscr{H}_{v}$ denotes the Hankel transform of order $v($ see $(2.7))$, and $\widetilde{C}_{p_{0}, q_{0}}^{j_{0}}\left(f_{\epsilon}^{\lambda}\right)(t):=$ $t^{-\left(p_{0}+q_{0}\right)} C_{p_{0}, q_{0}}^{j_{0}}\left(f_{\epsilon}^{\lambda}\right)(t)$. 
PRoof. In what follows $c_{\lambda}$ will stand for constants depending only on $\lambda$ and will vary from one line to another. Using Fact 2.2 we can rewrite $u_{\epsilon}^{\lambda}\left(\mathbf{z} ; s_{0}\right)$ as

$$
u_{\epsilon}^{\lambda}\left(\mathbf{z} ; s_{0}\right)=f_{\epsilon}^{\lambda} *_{\lambda} q_{i s_{0}}^{\lambda}(\mathbf{z})
$$

where

$$
q_{i s_{0}}^{\lambda}(\mathbf{z})=(4 \pi)^{-n}\left(\frac{\lambda}{i \sin \lambda s_{0}}\right)^{n} e^{(i / 4) \lambda\left(\operatorname{cotg} \lambda s_{0}\right)|\mathbf{z}|^{2}},
$$

which exits for all but countably many values of $\lambda$. Thus

$$
\begin{aligned}
\int_{S^{2 n-1}} & u_{\epsilon}^{\lambda}\left(r \omega ; s_{0}\right) \overline{Y_{p_{0}, q_{0}}^{j_{0}}(\omega)} d \sigma(\omega) \\
= & \int_{S^{2 n-1}}\left[\int_{\mathbb{C}^{n}} f_{\epsilon}^{\lambda}(r \omega-\mathbf{w}) q_{i s_{0}}^{\lambda}(\mathbf{w}) e^{i(\lambda / 2) \operatorname{Im}(r \omega \cdot \overline{\mathbf{w}})} d \mathbf{w}\right] \overline{Y_{p_{0}, q_{0}}^{j_{0}}(\omega)} d \sigma(\omega) \\
= & \int_{S^{2 n-1}}\left[\int_{\mathbb{C}^{n}} f_{\epsilon}^{\lambda}(\mathbf{w}) q_{i s_{0}}^{\lambda}(r \omega-\mathbf{w}) e^{-i(\lambda / 2) \operatorname{Im}(r \omega \cdot \overline{\mathbf{w}})} d \mathbf{w}\right] \overline{Y_{p_{0}, q_{0}}^{j_{0}}(\omega)} d \sigma(\omega) .
\end{aligned}
$$

We now expand $f_{\epsilon}^{\lambda}$ in terms of bigraded spherical harmonics as

$$
f_{\epsilon}^{\lambda}(t \eta)=\sum_{p, q \geq 0} \sum_{j=1}^{d(p, q)} C_{p, q}^{j}\left(f_{\epsilon}^{\lambda}\right)(t) Y_{p, q}^{j}(\eta)
$$

where $C_{p, q}^{j}\left(f_{\epsilon}^{\lambda}\right)$ is as in (2.4). Further, by [17, (2.8.7)],

$$
q_{i s_{0}}^{\lambda}(r \omega-t \eta)=(2 \pi)^{-n}|\lambda|^{n} \sum_{k=0}^{\infty} e^{-i(2 k+n)|\lambda| s_{0}} \varphi_{k, \lambda}^{(n-1)}(r \omega-t \eta),
$$

where $\varphi_{k, \lambda}^{(n-1)}$ is the Laguerre function given by (2.5). Now the Hecke-Bochner formula for the $\lambda$-twisted convolution (see Theorem 2.3) implies

$$
\begin{aligned}
\int_{0}^{\infty} & \int_{S^{2 n-1}} C_{p, q}^{j}\left(f_{\epsilon}^{\lambda}\right)(t) Y_{p, q}^{j}(\eta) \varphi_{k, \lambda}^{(n-1)}(r \omega-t \eta) e^{-i(\lambda / 2) r t \operatorname{Im}(\omega \cdot \bar{\eta})} t^{2 n-1} d t d \sigma(\eta) \\
& =\int_{0}^{\infty} \int_{S^{2 n-1}} \widetilde{C}_{p, q}^{j}\left(f_{\epsilon}^{\lambda}\right)(t) P_{p, q}^{j}(t \eta) \varphi_{k, \lambda}^{(n-1)}(r \omega-t \eta) e^{-i(\lambda / 2) r t \operatorname{Im}(\omega \cdot \bar{\eta})} t^{2 n-1} d t d \sigma(\eta) \\
& =\left[\widetilde{C}_{p, q}^{j}\left(f_{\epsilon}^{\lambda}\right) P_{p, q}^{j}\right] *_{-\lambda} \varphi_{k, \lambda}^{(n-1)}(r \omega) \\
& =(2 \pi)^{-n}|\lambda|^{p+q} P_{p, q}^{j}(r \omega)\left[\widetilde{C}_{p, q}^{j}\left(f_{\epsilon}^{\lambda}\right) *_{-\lambda} \varphi_{k-p, \lambda}^{(n+p+q-1)}\right](r \omega),
\end{aligned}
$$

where the convolution on the right-hand side is on $\mathbb{C}^{n+p+q}$. Here $P_{p, q}^{j}(r \omega):=$ ${ }^{p+q} Y_{p, q}^{j}(\omega)$ and $\widetilde{C}_{p, q}^{j}(f)(t):=t^{-(p+q)} C_{p, q}^{j}(f)(t)$. Above we have used the fact 
that $\varphi_{k, \lambda}^{(n-1)}=\varphi_{k,-\lambda}^{(n-1)}$. Using the orthogonality of the basis $\left\{Y_{p, q}^{j}: 1 \leq j \leq d(p, q)\right\}$,

$$
\begin{gathered}
\int_{S^{2 n-1}}\left[\int_{\mathbb{C}^{n}} f_{\epsilon}^{\lambda}(\mathbf{w}) q_{i s_{0}}^{\lambda}(r \omega-\mathbf{w}) e^{-i(\lambda / 2) \operatorname{Im}(r \omega \cdot \overline{\mathbf{w}})} d \mathbf{w}\right] \overline{Y_{p_{0}, q_{0}}^{j_{0}}(\omega)} d \sigma(\omega) \\
=c_{\lambda} r^{p_{0}+q_{0}} \sum_{k \geq p_{0}} e^{-i(2 k+n)|\lambda| s_{0}} \widetilde{C}_{p_{0}, q_{0}}^{j_{0}}\left(f_{\epsilon}^{\lambda}\right) *_{-\lambda} \varphi_{k-p_{0}, \lambda}^{\left(m_{0}\right)}(r \omega) \\
=c_{\lambda} r^{p_{0}+q_{0}} \sum_{k=0}^{\infty} e^{-i\left(2 k+n+2 p_{0}\right)|\lambda| s_{0}} \widetilde{C}_{p_{0}, q_{0}}^{j_{0}}\left(f_{\epsilon}^{\lambda}\right) *_{-\lambda} \varphi_{k, \lambda}^{\left(m_{0}\right)}(r \omega),
\end{gathered}
$$

where

$$
m_{0}:=n+p_{0}+q_{0}-1
$$

On the other hand, by (2.6),

$$
\begin{aligned}
\widetilde{C}_{p_{0}, q_{0}}^{j_{0}}\left(f_{\epsilon}^{\lambda}\right) *_{-\lambda} \varphi_{k, \lambda}^{\left(m_{0}\right)}(r \omega)=c_{\lambda} & \frac{\Gamma(k+1)}{\Gamma\left(k+m_{0}+1\right)} \varphi_{k, \lambda}^{\left(m_{0}\right)}(r \omega) \\
& \times\left(\int_{0}^{\infty} \widetilde{C}_{p_{0}, q_{0}}^{j_{0}}\left(f_{\epsilon}^{\lambda}\right)(t) \varphi_{k, \lambda}^{\left(m_{0}\right)}(t) t^{2 m_{0}+1} d t\right)
\end{aligned}
$$

Hence

$$
\begin{aligned}
\int_{S^{2 n-1}} & u_{\epsilon}^{\lambda}\left(r \omega ; s_{0}\right) \overline{Y_{p_{0}, q_{0}}^{j_{0}}(\omega)} d \sigma(\omega) \\
= & c_{\lambda} r^{p_{0}+q_{0}} \sum_{k=0}^{\infty} \frac{\Gamma(k+1)}{\Gamma\left(k+m_{0}+1\right)} e^{-i\left(2 k+n+2 p_{0}\right)|\lambda| s_{0}} e^{-(|\lambda| / 4) r^{2}} \\
& \times L_{k}^{\left(m_{0}\right)}\left(\frac{|\lambda|}{2} r^{2}\right)\left(\int_{0}^{\infty} \widetilde{C}_{p_{0}, q_{0}}^{j_{0}}\left(f_{\epsilon}^{\lambda}\right)(t) \varphi_{k, \lambda}^{\left(m_{0}\right)}(t) t^{2 m_{0}+1} d t\right) \\
= & c_{\lambda} r^{p_{0}+q_{0}} \int_{0}^{\infty} \widetilde{C}_{p_{0}, q_{0}}^{j_{0}}\left(f_{\epsilon}^{\lambda}\right)(t) K_{\lambda}\left(r, t ; s_{0}\right) t^{2 m_{0}+1} d t
\end{aligned}
$$

with

$$
\begin{aligned}
& K_{\lambda}\left(r, t ; s_{0}\right):=e^{-(|\lambda| / 4)\left(r^{2}+t^{2}\right)} \sum_{k=0}^{\infty} \frac{\Gamma(k+1)}{\Gamma\left(k+m_{0}+1\right)} \\
& \times e^{-i\left(2 k+n+2 p_{0}\right)|\lambda| s_{0}} L_{k}^{\left(m_{0}\right)}\left(\frac{|\lambda|}{2} r^{2}\right) L_{k}^{\left(m_{0}\right)}\left(\frac{|\lambda|}{2} t^{2}\right) .
\end{aligned}
$$

Now we can use the following Hille-Hardy identity (see, for instance, [16])

$$
\sum_{k=0}^{\infty} \frac{\Gamma(k+1)}{\Gamma(k+v+1)} L_{k}^{(v)}(x) L_{k}^{(v)}(y) w^{k}=\frac{e^{-(w /(1-w))(x+y)}}{(1-w)^{(v+1)}} J_{v}\left(\frac{2(-x y w)^{1 / 2}}{1-w}\right),
$$

where $J_{v}$ is the normalized Bessel function (2.8). Thus we may rewrite the kernel $K_{\lambda}$ as

$$
K_{\lambda}\left(r, t ; s_{0}\right)=\frac{e^{i|\lambda| s_{0}\left(q_{0}-p_{0}\right)}}{\left(2 i \sin \left(|\lambda| s_{0}\right)\right)^{m_{0}+1}} e^{i(\lambda / 4)\left(r^{2}+t^{2}\right) \operatorname{cotg}\left(\lambda s_{0}\right)} J m_{0}\left(\frac{\lambda}{2} \frac{r t}{\sin \left(\lambda s_{0}\right)}\right) .
$$


Thus we arrive at

$$
\begin{aligned}
\int_{S^{2 n-1}} & u_{\epsilon}^{\lambda}\left(r \omega ; s_{0}\right) \overline{Y_{p_{0}, q_{0}}^{j_{0}}(\omega)} d \sigma(\omega) \\
= & c_{\lambda} r^{p_{0}+q_{0}} \int_{0}^{\infty} e^{i(\lambda / 4)\left(r^{2}+t^{2}\right) \operatorname{cotg}\left(\lambda s_{0}\right)} \widetilde{C}_{p_{0}, q_{0}}^{j_{0}}\left(f_{\epsilon}^{\lambda}\right)(t) J_{m_{0}}\left(\frac{\lambda}{2} \frac{r t}{\sin \left(\lambda s_{0}\right)}\right) t^{2 m_{0}+1} d t \\
= & c_{\lambda} r^{p_{0}+q_{0}} e^{i(\lambda / 4) r^{2} \operatorname{cotg}\left(\lambda s_{0}\right)} \mathscr{H}_{m_{0}}\left(e^{i(\lambda / 4)(\cdot)^{2} \operatorname{cotg}\left(\lambda s_{0}\right)} \widetilde{C}_{p_{0}, q_{0}}^{j_{0}}\left(f_{\epsilon}^{\lambda}\right)\right)\left(\frac{\lambda r}{2 \sin \left(\lambda s_{0}\right)}\right) .
\end{aligned}
$$

Hence Theorem 3.4 is proven.

Now we are ready to complete the proof of Theorem 3.1.

The estimate (3.2a) on $f_{\epsilon}(\mathbf{z}, t)$ together with Fact 2.1 lead us to

$$
\left|f_{\epsilon}^{\lambda}(\mathbf{z})\right| \lesssim e^{-(1 / 4)|\mathbf{z}|^{2} /(\alpha+\epsilon)} .
$$

Thus, the spherical harmonic coefficient $\widetilde{C}_{p_{0}, q_{0}}^{j_{0}}\left(f_{\epsilon}^{\lambda}\right)$ satisfies

$$
\left|\widetilde{C}_{p_{0}, q_{0}}^{j_{0}}\left(f_{\epsilon}^{\lambda}\right)(t)\right| \lesssim t^{-\left(p_{0}+q_{0}\right)} e^{-(1 / 4) t^{2} /(\alpha+\epsilon)}
$$

On the other hand, by means of Theorem 3.4 and the estimate (3.2b) on $u_{\epsilon}\left(\mathbf{z}, t ; s_{0}\right)$, we deduce that

$$
\left|\mathscr{H}_{n+p_{0}+q_{0}-1}\left(e^{i(\lambda / 4)(\cdot)^{2} \operatorname{cotg}\left(\lambda s_{0}\right)} \widetilde{C}_{p_{0}, q_{0}}^{j_{0}}\left(f_{\epsilon}^{\lambda}\right)\right)\left(\frac{\lambda r}{2 \sin \left(\lambda s_{0}\right)}\right)\right| \leq c_{\lambda} r^{-\left(p_{0}+q_{0}\right)} e^{-(1 / 4) r^{2} /(\beta+\epsilon)} .
$$

That is,

$$
\left|\mathscr{H}_{n+p_{0}+q_{0}-1}\left(e^{i(\lambda / 4)(\cdot)^{2} \operatorname{cotg}\left(\lambda s_{0}\right)} \widetilde{C}_{p_{0}, q_{0}}^{j_{0}}\left(f_{\epsilon}^{\lambda}\right)\right)(r)\right| \leq c_{\lambda} r^{-\left(p_{0}+q_{0}\right)} e^{-(1 / 4)\left(2 \sin \left(\lambda s_{0}\right) / \lambda s_{0}\right)^{2} r^{2} s_{0}^{2} /(\beta+\epsilon)} .
$$

Given $\alpha, \beta>0$ such that $\alpha \beta<s_{0}^{2}$ we can choose $\epsilon>0$ in such a way that $(\alpha+\epsilon)$ $(\beta+\epsilon)<s_{0}^{2}$. We can also choose $\delta>0$ small enough so that for $0<\lambda<\delta$ we have $(\alpha+\epsilon)(\beta+\epsilon)<s_{0}^{2}\left(\sin \left(\lambda s_{0}\right) / \lambda s_{0}\right)^{2}$. This inequality can be written as

$$
\frac{1}{4(\alpha+\epsilon)} \frac{s_{0}^{2}}{4(\beta+\epsilon)}\left(\frac{2 \sin \left(\lambda s_{0}\right)}{\lambda s_{0}}\right)^{2}>\frac{1}{4} .
$$

Therefore, by Hardy's theorem for the Hankel transform (see Theorem 2.4), we deduce that for $0<\lambda<\delta$ we have $\widetilde{C}_{p_{0}, q_{0}}^{j_{0}}\left(f_{\epsilon}^{\lambda}\right)=0$, for all $p_{0}, q_{0} \geq 0$ and for all $1 \leq j_{0} \leq$ $d\left(p_{0}, q_{0}\right)$. That is, $f_{\epsilon}^{\lambda}=0$ on $\mathbb{C}^{n}$ for $0<\lambda<\delta$. By the remark stated after Lemma 3.3, we deduce that $f_{\epsilon}^{\lambda}=0$ for all $\lambda \in \mathbb{C}$, and thus $f_{\epsilon}=0$ on $\mathbb{H}^{n}$. That is, $f=0$ on $\mathbb{H}^{n}$. This finishes the proof Theorem 3.1.

\section{Proof of Theorem 1.2}

Let $\mathfrak{g}$ be a two step nilpotent Lie algebra over $\mathbb{R}$ with an inner product $\langle\cdot, \cdot\rangle$. The corresponding simply connected Lie group is denoted by $G$. Let $z$ be the center of 
$\mathfrak{g}$ and $\mathfrak{v}$ the orthogonal complement of $\mathfrak{z}$ in $\mathfrak{g}$. The Lie algebra $\mathfrak{g}$ is called an $H$-type algebra if for every $\mathbf{v} \in \mathfrak{v}$, the $\operatorname{map}_{\mathbf{v}}: \mathfrak{v} \rightarrow \mathfrak{z}$ is a surjective isometry when restricted to the orthogonal complement of its kernel.

For the $H$-type algebra $\mathfrak{g}=\mathfrak{v} \oplus \jmath$, let $\operatorname{dim}(\mathfrak{v})=2 n$ and $\operatorname{dim}(\mathfrak{\jmath})=k$. The class of groups of $H$-type includes the Heisenberg group $\mathbb{H}^{n}$ when $k=1$. Let $\eta$ be a unit element in $z$ and denote its orthogonal complement in $z$ by $\eta^{\perp}$. The quotient algebra $\mathfrak{g} / \eta^{\perp}$ is a Lie algebra with Lie bracket $[X, Y]_{\eta}=\langle[X, Y], \eta\rangle$.

The quotient $\mathrm{g} / \eta^{\perp}$ is an $H$-type algebra with inner product $\langle\cdot, \cdot\rangle_{\eta}$ given by $\left\langle\left(\mathbf{v}_{1}, t_{1}\right),\left(\mathbf{v}_{2}, t_{2}\right)\right\rangle_{\eta}=\left\langle\mathbf{v}_{1}, \mathbf{v}_{2}\right\rangle+t_{1} t_{2}$, where $\mathbf{v}_{1}, \mathbf{v}_{2} \in \mathfrak{v}, t_{1}, t_{2} \in \mathbb{R}$, and $\left\langle\mathbf{v}_{1}, \mathbf{v}_{2}\right\rangle$ is the inner product in $\mathfrak{g}$. Here $(\mathbf{v}, t)$ stands for the coset of $\mathbf{v}+t \eta$ in $\mathfrak{g} / \eta^{\perp}$. Moreover, if we denote by $G_{\eta}$ the simply connected Lie group with Lie algebra $\mathfrak{g} / \eta^{\perp}$, then by [14], the Lie group $G_{\eta}$ is isomorphic to the Heisenberg group $\mathbb{H}^{n}=\mathbb{C}^{n} \times \mathbb{R}$. We refer to [1] for more details on the theory of $H$-type groups.

We fix an orthonormal basis $X_{1}, \ldots, X_{2 n}$ for $\mathfrak{v}$, and define the sub-Laplacian by

$$
\mathcal{L}_{G}=-\sum_{j=1}^{2 n} X_{j}^{2}
$$

It is known that $\mathcal{L}_{G}$ generates a semigroup which is given by convolution with the heat kernel for $G$. As in the case of the Heisenberg group, the kernel is explicitly known and is given by

$$
h_{s}(\mathbf{v}, \mathbf{t})=\frac{2}{(4 \pi)^{n+k / 2}} \int_{0}^{\infty} J_{k / 2-1}(\lambda|\mathbf{t}|)\left(\frac{\lambda}{\sinh (s \lambda)}\right)^{n} e^{-\frac{1}{4} \lambda(\operatorname{coth} s \lambda)|\mathbf{v}|} \lambda^{k-1} d \lambda,
$$

for $(\mathbf{v}, \mathbf{t}) \in G$ and $s>0$. Here $J_{v}$ denotes the normalized Bessel function (2.8). This formula has been proved in [13], where the author also obtains the integral expression for the analytic continuation, $h_{\zeta}$, of the heat kernel $h_{s}$ as long as $\operatorname{Rel}(\zeta)>0$.

Now we consider the solution of the Schrödinger equation on $G \times \mathbb{R}$

$$
\left\{\begin{array}{l}
i \partial_{s} u(\mathbf{v}, \mathbf{t} ; s)=\mathcal{L}_{G} u(\mathbf{v}, \mathbf{t} ; s), \quad(\mathbf{v}, \mathbf{t}) \in G, s \in \mathbb{R} \\
u(\mathbf{v}, \mathbf{t} ; 0)=f(\mathbf{v}, \mathbf{t}),
\end{array}\right.
$$

which is given by $u(\mathbf{v}, \mathbf{t} ; s)=e^{-i s \mathcal{L}_{G}} f(\mathbf{v}, \mathbf{t})$. When we replace the initial condition $f$ by $e^{-\epsilon \mathcal{L}_{G}} f$, for some $\epsilon>0$, then the solution is given by

$$
u_{\epsilon}(\mathbf{v}, \mathbf{t} ; s)=f * h_{\zeta}(\mathbf{v}, \mathbf{t}) \quad \text { with } \quad \zeta=\epsilon+i s .
$$

We claim that the uniqueness Theorem 3.1 for the Schrödinger equation on $\mathbb{H}^{n} \times \mathbb{R}$ holds true in the more general setting $G \times \mathbb{R}$. The rest of this section is devoted to the proof of Theorem 1.2.

For a suitable function $f$ on $G$ we define its partial Radon transform $\mathcal{R}_{\eta} f(\mathbf{v}, t)$ on $G_{\eta}$ by

$$
\mathcal{R}_{\eta} f(\mathbf{v}, t)=\int_{\eta^{\perp}} f(\mathbf{v}, t \eta+v) d v
$$


where $d v$ is the Lebesgue measure on $\eta^{\perp}$. Since $G_{\eta}$ can be identified with the Heisenberg group $\mathbb{H}^{n}$, we can think of $\mathcal{R}_{\eta} f$ as a function on $\mathbb{H}^{n}$. With this identification, it has been proved in [13] that $\mathcal{R}_{\eta} h_{s}(\mathbf{v}, t)=q_{s}(\mathbf{v}, t)$, for $s>0$, where $q_{s}(\mathbf{v}, t)$ is the heat kernel from Section 2. The latter identity between the heat kernels holds true even when $s$ is complex with $\operatorname{Rel}(s)>0$.

In view of the assumptions on $f(\mathbf{v}, \mathbf{t})$ and $u\left(\mathbf{v}, \mathbf{t} ; s_{0}\right)$, it follows that $\mathcal{R}_{\eta} f(\mathbf{v}, t)$ and $\mathcal{R}_{\eta} u\left(\mathbf{v}, t ; s_{0}\right)$ satisfy

$$
\begin{array}{r}
\left|\mathcal{R}_{\eta} f(\mathbf{v}, t)\right| \lesssim q_{\alpha}(\mathbf{v}, t), \\
\left|\mathcal{R}_{\eta} u\left(\mathbf{v}, t ; s_{0}\right)\right| \lesssim q_{\beta}(\mathbf{v}, t) .
\end{array}
$$

Moreover, using the fact that under the Radon transform $\mathcal{R}_{\eta}$, the sub-Laplacian $\mathcal{L}_{G}$ goes into the sub-Laplacian $\mathcal{L}_{\mathbb{H}^{n}}$ (see [14]), it follows that $\mathcal{R}_{\eta} u$ solves the Schrödinger equation on $\mathbb{H}^{n} \times \mathbb{R}$ with initial data $\mathcal{R}_{\eta} f(\mathbf{v}, t)$. Hence we can appeal to Theorem 3.1 to conclude that $\mathcal{R}_{\eta} u(\mathbf{v}, t ; s)=0$ for all $s \in \mathbb{R}$ and for all $\eta \in z$ whenever $\alpha \beta<s_{0}^{2}$. Now the injectivity of the Radon transform implies that if $\alpha \beta<s_{0}^{2}$, then $u(\mathbf{v}, \mathbf{t} ; s)=0$ for all $(\mathbf{v}, \mathbf{t}) \in G$ and $s \in \mathbb{R}$. This establishes Theorem 1.2.

\section{The Grushin operator}

The spectral decomposition of the Grushin operator $\mathcal{G}=-\Delta_{\mathbb{R}^{n}}-|x|^{2} \partial_{t}^{2}$ is given by

$$
\mathcal{G} f(x, t)=(2 \pi)^{-1} \int_{-\infty}^{\infty} e^{-i \lambda t}\left(\sum_{k=0}^{\infty}(2 k+n)|\lambda| P_{k}(\lambda) f^{\lambda}(x)\right) d \lambda,
$$

where $P_{k}(\lambda)$ are the spectral projections of the scaled Hermite operator $H(\lambda):=$ $-\Delta_{\mathbb{R}^{n}}+\lambda^{2}|x|^{2}$ so that

$$
H(\lambda)=\sum_{k=0}^{\infty}(2 k+n)|\lambda| P_{k}(\lambda)
$$

and $f^{\lambda}$ is as in (2.2). The heat kernel associated to $\mathcal{G}$ is given by

$$
\kappa_{s}(x, y, t)=(2 \pi)^{-1} \int_{-\infty}^{\infty} \Gamma_{\lambda}(x, y, s) e^{-i t \lambda} d \lambda,
$$

where $\Gamma_{\lambda}(x, y, s)$ is the well-known heat kernel associated to the Hermite operator $H(\lambda)$ (see, for instance, [16]):

$$
\Gamma_{\lambda}(x, y, s)=c_{n}\left(\frac{\lambda}{\sinh 2 \lambda s}\right)^{n / 2} e^{-\left(|x|^{2}+|y|^{2}\right)(\lambda / 2) \operatorname{coth} 2 \lambda s+(2 / \sinh 2 \lambda s) x \cdot y} .
$$

The solution of the heat equation associated to $\mathcal{G}$ is given by

$$
e^{-s \mathcal{G}} f(x, t)=\int_{\mathbb{R}^{n+1}} \kappa_{s}\left(x, y, t-t^{\prime}\right) f\left(y, t^{\prime}\right) d y d t^{\prime} .
$$


Note that, due to the zeros of the hyperbolic sine function appearing in the expression of $\Gamma_{\lambda}(x, y, s)$, the kernel $\kappa_{s}(x, y, t)$ cannot be analytically continued for purely imaginary values of $s$. However, as in the case of the sub-Laplacian $\mathcal{L}_{G}$, the kernel $\kappa_{\epsilon+i s}(x, y, t)$ is well defined for all $\epsilon>0$ and the function

$$
u_{\epsilon}(x, t ; s):=\int_{\mathbb{R}^{n+1}} \kappa_{\epsilon+i s}\left(x, y, t-t^{\prime}\right) f\left(y, t^{\prime}\right) d y d t^{\prime}
$$

solves the Schrödinger equation

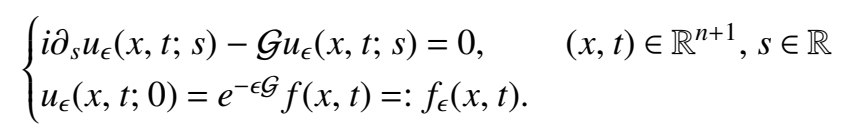

The heat kernel $\kappa_{s}(x, y, t)$ satisfies the semigroup property

$$
\int_{\mathbb{R}^{n+1}} \kappa_{\alpha}\left(x, z, t-t^{\prime}\right) \kappa_{\beta}\left(z, y, t^{\prime}\right) d z d t^{\prime}=\kappa_{\alpha+\beta}(x, y, t) .
$$

Therefore, when $f$ and $u$ are as in Theorem 1.3 then we have the estimates

$$
\left|f_{\epsilon}(x, t)\right| \lesssim \kappa_{\alpha+\epsilon}(x, 0, t), \quad\left|u_{\epsilon}\left(x, t ; s_{0}\right)\right| \lesssim \kappa_{\beta+\epsilon}(x, 0, t) .
$$

Hence we will be working with $f_{\epsilon}$ and $u_{\epsilon}$ instead of $f$ and $u$.

Following the same strategy used in the proof of Theorem 3.1, we reduce the proof of Theorem 1.3 to Hardy's theorem for the Hankel transform. To do that we need the Hecke-Bochner formula for the Hermite projection operators $P_{k}(\lambda)$. For each integer $p$, we define $\mathcal{P}_{p}$ to be the space of all polynomials on $\mathbb{R}^{n}$ of the form $\sum_{|\alpha|=p} a_{\alpha} x^{\alpha}$. Let $\mathcal{H}_{p}:=\left\{P \in \mathcal{P}_{p}: \Delta P=0\right\}$, where $\Delta$ denotes the Laplacian on $\mathbb{R}^{n}$. The elements of $\mathcal{H}_{p}$ are called solid harmonics of degree $p$. We will denote by $\mathcal{S}_{p}$ the space of all restrictions of solid harmonics of degree $p$ to the sphere $S^{n-1}$. Then it is well known that the space $L^{2}\left(S^{n-1}\right)$ is the orthogonal direct sum of the spaces $\mathcal{S}_{p}$, with $p \geq 0$. We choose an orthonormal basis $\left\{Y_{p}^{j} \mid 1 \leq j \leq d(p)\right\}$ for $\mathcal{S}_{p}$.

Theorem 5.1. Let $p_{0} \geq 0$ and $1 \leq j_{0} \leq d\left(p_{0}\right)$ be fixed integers. Then

$$
\begin{aligned}
\int_{S^{n-1}} & u_{\epsilon}^{\lambda}\left(r \omega ; s_{0}\right) \overline{Y_{p_{0}}^{j_{0}}(\omega)} d \sigma(\omega)=c_{\lambda} e^{-i(\lambda / 2) \operatorname{coth} 2 \lambda s_{0} r^{2}} \\
& \times \mathscr{H}_{(n / 2)+p_{0}-1}\left(e^{-i(\lambda / 2) \operatorname{coth} 2 \lambda(\cdot)^{2}} C_{p_{0}}^{j_{0}}\left(f_{\epsilon}^{\lambda}\right)\right)\left(\frac{\lambda r}{\sinh 2 \lambda s_{0}}\right),
\end{aligned}
$$

where $u_{\epsilon}^{\lambda}\left(x ; s_{0}\right)$ is the inverse Fourier transform of $u_{\epsilon}\left(x, t ; s_{0}\right)$ with respect to $t, C_{p_{0}}^{j_{0}}\left(f_{\epsilon}^{\lambda}\right)$ denotes the spherical harmonic coefficient of $f_{\epsilon}^{\lambda}$ with respect to $Y_{p_{0}}^{j_{0}}$, and $c_{\lambda}$ is a constant which depends only on $\lambda$.

In order to prove the above theorem we make use of the following Hecke-Bochner formula for the Hermite projection operators, see [16]. 
Theorem 5.2. Let $\psi(x)=F(|x|) P(x)$, where $P$ is a solid harmonic of degree $m$. Then for $|w|<1$,

$$
\begin{aligned}
& \sum_{k=0}^{\infty} P_{k}(\lambda) \psi(x) w^{k} \\
&=2 i^{-((n / 2)+m-1)}\left(1-w^{2}\right)^{-1} w^{-((n / 2)-1)} r^{-((n / 2)+m-1)} \\
& \times\left(\int_{0}^{\infty} F(s) e^{-(|\lambda| / 2)\left(\left(1+w^{2}\right) /\left(1-w^{2}\right)\right) s^{2}} J_{(n / 2)+m-1}\left(\frac{2 i w|\lambda|}{1-w^{2}} r s\right) s^{(n / 2)+m} d s\right) \\
& \times e^{-(|\lambda| / 2)\left(\left(1+w^{2}\right) /\left(1-w^{2}\right)\right) r^{2}} P(x),
\end{aligned}
$$

where $r=|x|$ and $J_{\alpha}$ is the Bessel function of order $\alpha>-1$.

Theorem 5.1 is proved using the above formula as in the case of the sub-Laplacian $\mathcal{L}_{\mathbb{H}^{n}}$. We omit the details. Once Theorem 5.1 is proved, the uniqueness theorem for the Grushin operator follows immediately from Hardy's Theorem 2.4 for the Hankel transform.

\section{Some concluding remarks}

It would be interesting to see if Theorem 3.1 is sharp. Though we believe it is sharp, we are not able to prove it. The main reason for the difficulty lies in the fact that the heat kernel $q_{s}(\mathbf{z}, t)$ on $\mathbb{H}^{n}$ does not have Gaussian decay in the central variable. For the same reason the equality case of Hardy's theorem for the group Fourier transform on the Heisenberg group is still an open problem. However, if we assume conditions on $f^{\lambda}$ and $u^{\lambda}$ instead of on $f$ and $u$ we can prove the following result.

THeorem 6.1. Let $u(\mathbf{z}, t ; s)$ be the solution to the Schrödinger equation for the subLaplacian $\mathcal{L}$ on $\mathbb{H}^{n}$ with initial condition $f$. Fix $\lambda \neq 0$ and suppose that

$$
\left|f^{\lambda}(\mathbf{z})\right| \lesssim q_{\alpha}^{\lambda}(\mathbf{z}), \quad\left|u^{\lambda}\left(\mathbf{z} ; s_{0}\right)\right| \lesssim q_{\beta}^{\lambda}(\mathbf{z}),
$$

for some $\alpha, \beta>0$ and for a fixed $s_{0} \in \mathbb{R}^{*}$. Then there exists a constant $c_{\lambda}$ which depends only on $\lambda$ such that

$$
f^{\lambda}(\mathbf{z})=c_{\lambda} q_{\alpha}^{\lambda}(\mathbf{z}) e^{-i(\lambda / 4)|\mathbf{z}|^{2} \operatorname{cotg}\left(\lambda s_{0}\right)}
$$

whenever $\tanh (\alpha \lambda) \tanh (\beta \lambda)=\sin ^{2}\left(\lambda s_{0}\right)$.

To prove this theorem, we can proceed as in the proof of Theorem 3.1. We end up with the estimates

$$
\left|\mathscr{H}_{m_{0}}\left(e^{i(\lambda / 4)(\cdot)^{2} \operatorname{cotg}\left(\lambda s_{0}\right)} \widetilde{C}_{p_{0}, q_{0}}^{j_{0}}\left(f^{\lambda}\right)\right)(r)\right| \leq c_{\lambda} r^{-\left(p_{0}+q_{0}\right)} e^{-(\lambda / 4) \operatorname{coth}(\lambda \beta)\left(2 \sin \left(\lambda s_{0}\right) / \lambda s_{0}\right)^{2} r^{2}},
$$

with $m_{0}:=n+p_{0}+q_{0}-1$, and

$$
\left|\widetilde{C}_{p_{0}, q_{0}}^{j_{0}}\left(f^{\lambda}\right)(r)\right| \leq c_{\lambda} e^{-(\lambda / 4) \operatorname{coth}(\lambda \alpha) r^{2}} .
$$


We can now appeal to the equality case of Hardy's theorem for the Hankel transform (Theorem 2.4) to conclude that there exists a constant $c_{\lambda}\left(p_{0}, q_{0}, j_{0}\right)$ such that

$$
C_{p_{0}, q_{0}}^{j_{0}}\left(f^{\lambda}\right)(r)=c_{\lambda}\left(p_{0}, q_{0}, j_{0}\right) r^{p_{0}+q_{0}} e^{-(\lambda / 4) \operatorname{coth}(\lambda \alpha) r^{2}} e^{-i(\lambda / 4) r^{2} \operatorname{cotg}\left(\lambda s_{0}\right)} .
$$

But this is not compatible with the hypothesis on $f^{\lambda}$ unless $c_{\lambda}\left(p_{0}, q_{0}, j_{0}\right)=0$ for all $\left(p_{0}, q_{0}\right) \neq(0,0)$. Hence $f^{\lambda}$ is radial and equals $c_{\lambda} q_{\alpha}^{\lambda}(\mathbf{z}) e^{-i(\lambda / 4)|\mathbf{z}|^{2} \operatorname{cotg}\left(\lambda s_{0}\right)}$. This proves Theorem 6.1.

The above result can be viewed as a uniqueness theorem for solutions of the Schrödinger equation associated to the twisted Laplacian $\mathcal{L}_{\lambda}$ defined via the identity $\mathcal{L}_{\mathbb{H}^{n}}\left(e^{i \lambda t} f(\mathbf{z})\right)=e^{i \lambda t} \mathcal{L}_{\lambda} f(\mathbf{z})$. Indeed, $q_{\alpha}^{\lambda}(\mathbf{z})$ is the heat kernel associated to this operator. We refer to [17, (2.3.7)] for the explicit expression of $\mathcal{L}_{\lambda}$. We can also consider Theorem 6.1 as an analogue of Hardy's theorem for fractional powers of the symplectic Fourier transform. In fact, the unitary operator $e^{i n s} e^{-i s \mathcal{L}_{1}}$ with $s=\pi / 2$ is just the symplectic Fourier transform. Thus, the above theorem for $s_{0}=\pi / 2$ follows immediately from Hardy's theorem for the Euclidean Fourier transform, whereas for other values of $s_{0}$ we require a long-winding proof.

For the sake of completeness we state another result which can be considered as a theorem for the fractional Fourier transform as well as a theorem for solutions of the Schrödinger equation associated to the Hermite operator $H=-\Delta+|x|^{2}$ on $\mathbb{R}^{n}$. This elliptic operator generates the Hermite semigroup, whose kernel is known explicitly. We also know that $e^{(i / 4) n \pi} e^{-(i / 4) \pi H}$ is the Fourier transform on $\mathbb{R}^{n}$.

THEOREM 6.2. Let $u(x, s)=e^{-i s H} f(x)$ be the solution to the Schrödinger equation

$$
i \partial_{s} u(x, s)-H u(x, s)=0, \quad u(x, 0)=f(x) .
$$

Suppose that

$$
|f(x)|=O\left(e^{-\alpha|x|^{2}}\right), \quad\left|u\left(x, s_{0}\right)\right|=O\left(e^{-\beta|x|^{2}}\right)
$$

for some $\alpha, \beta>0$. Then $u \equiv 0$ on $\mathbb{R}^{n} \times \mathbb{R}$ whenever $\alpha \beta \sin ^{2}\left(2 s_{0}\right)>\frac{1}{4}$.

The theorem follows from the Euclidean Hardy's theorem for $\mathbb{R}^{n}$ once we realize $u$ as the Fourier transform of a function. But this is easy to check in view of the Mehler's formula for the Hermite functions (see [17]). Indeed, in view of this formula, the kernel of $e^{-i s H}$ is given by

$$
K_{r}(x, y)=\pi^{-n / 2}\left(1-r^{2}\right)^{-n / 2} e^{-(1 / 2)\left(\left(1+r^{2}\right) /\left(1-r^{2}\right)\right)\left(|x|^{2}+|y|^{2}\right)+\left(2 r /\left(1-r^{2}\right)\right) x \cdot y},
$$

where $r=e^{-2 i s}$. Therefore, the solution $u(x, s)$ can be written as

$$
u(x, s)=e^{-i s H} f(x)=c_{n, s} e^{(i / 2)|x|^{2} \cot (2 s)} \widehat{g_{s}}\left(\frac{1}{\sin (2 s)} y\right),
$$

where $g_{s}(x):=e^{(i / 2)|x|^{2} \cot (2 s)} f(x)$ and ' - , stands for the Euclidean Fourier transform on $\mathbb{R}^{n}$. The assumptions on $f$ and $u$ translate into

$$
\left|g_{s_{0}}(x)\right| \lesssim e^{-\alpha|x|^{2}}, \quad\left|\widehat{g_{s_{0}}}(y)\right| \lesssim e^{-\beta|y|^{2} \sin ^{2}\left(2 s_{0}\right)},
$$

and hence Theorem 6.2 follows from the Euclidean Hardy's theorem. 
We conclude this paper with one more remark. If we use other uncertainty principles such as Beurling's theorem or Benedick's theorem in place of Hardy's theorem we can obtain different versions of uniqueness theorems for the solutions of Schrödinger equations. For example, after this paper has been submitted for publication, in [12] the authors have proved a uniqueness theorem (for symmetric spaces) under a condition of Beurling type on $f$ and $u$. We restrain from stating such results as the proofs do not involve any new ideas.

\section{Acknowledgements}

This work was done while the second author was visiting Universite de Lorraine, Nancy. He wishes to thank Salem Ben Said for the invitation and the warm hospitality during his visit.

\section{References}

[1] J. Berndt, F. Tricerri and L. Vanhecke, Generalized Heisenberg Groups and Damek-Ricci Harmonic Spaces, Lecture Notes in Mathematics, 1598 (Springer, Berlin, 1995).

[2] S. Chanillo, 'Uniqueness of solutions to Schrödinger equations on complex semi-simple Lie groups', Proc. Indian Acad. Sci. Math. Sci. 117 (2007), 325-331.

[3] M. Cowling, L. Escauriaza, C. E. Kenig, G. Ponce and L. Vega, 'The Hardy uncertainty principle revisted', Indiana Univ. Math. J. 59(6) (2010), 2007-2026.

[4] L. Escauriaza, C. E. Kenig, G. Ponce and L. Vega, 'Hardy's uncertainty principle, convexity and Schrödinger evolutions', J. Eur. Math. Soc. (JEMS) 10 (2008), 883-907.

[5] L. Escauriaza, C. E. Kenig, G. Ponce and L. Vega, 'Convexity of free solutions of Schrödinger equations with Gaussian decay', Math. Res. Lett. 15 (2008), 957-971.

[6] L. Escauriaza, C. E. Kenig, G. Ponce and L. Vega, 'The sharp Hardy uncertainty principle for Schrödinger evolutions', Duke Math. J. 155 (2010), 163-187.

[7] L. Escauriaza, C. E. Kenig, G. Ponce and L. Vega, 'Uncertainty principles of Morgan type and Schrödinger evolutions', J. Lond. Math. Soc. (2) 83 (2011), 187-207.

[8] I. D. Ionescu and C. E. Kenig, 'Uniqueness properties of solutions of Schrödinger equations', J. Funct. Anal. 232 (2006), 90-136.

[9] A. Kaplan, 'Fundamental solutions for a class of hypoelliptic PDE generated by composition of quadratic forms', Trans. Amer. Math. Soc. 258 (1980), 147-153.

[10] C. E. Kenig, G. Ponce and L. Vega, 'On the support of solutions of nonlinear Schrödinger equations', Comm. Pure Appl. Math. 56 (2002), 1247-1262.

[11] J. Ludwig and D. Müller, 'Uniqueness of solutions to Schrödinger equations on 2-step nilpotent Lie groups', 2012, arXiv:1207.4652.

[12] A. Pasquale and M. Sundari, 'Uncertainty principles for the Schrödinger equation on Riemannian symmetric spaces of the noncompact type', Ann. Inst. Fourier (Grenoble) 62 (2012), 859-886.

[13] J. Randall, 'The heat kernel for generalized Heisenberg groups', J. Geom. Anal. 6 (1996), 287-316.

[14] F. Ricci, 'Commutative algebras of invariant functions on groups of Heisenberg type', J. Lond. Math. Soc. 32 (1985), 265-271.

[15] L. Robbiano, 'Unicité forte à l'infini pour KdV', ESAIM Control Optim. Calc. Var. 8 (2002), 933-939.

[16] S. Thangavelu, Lectures on Hermite and Laguerre expansions, Mathematical Notes, 42 (Princeton University Press, Princeton, 1993).

[17] S. Thangavelu, An Introduction to the Uncertainty Principle. Hardy's Theorem on Lie Groups, Progress in Mathematics, 217 (Birkhäuser, Boston, MA, 2004). 
[18] V.-K. Tuan, 'Uncertainty principles for the Hankel transform', Integral Transforms Spec. Funct. 18 (2007), 369-381.

[19] B.-Y. Zhang, 'Unique continuation for the Korteweg-de Vries equation', SIAM J. Math. Anal. 23 (1992), 55-71.

SALEM BEN SAÏD, Institut Elie Cartan, Université de Lorraine, B.P. 239, 54506 Vandoeuvre-Les-Nancy, France e-mail: Salem.BenSaid@univ-lorraine.fr

SUNDARAM THANGAVELU, Department of Mathematics, Indian Institute of Science, Bangalore 560 012, India e-mail: veluma@math.iisc.ernet.in

VENKU NAIDU DOGGA, Department of Mathematics, Indian Institute of Science, Bangalore 560 012, India e-mail: venku11@math.iisc.ernet.in 